OPEN ACCESS

Edited by:

Mina Luqing $X u$,

Yale University, United States

Reviewed by:

Muhammad Bilal Abid,

Medical College of Wisconsin,

United States

Somsubhra Nath,

Saroj Gupta Cancer Centre and

Research Institute, India

${ }^{*}$ Correspondence:

Jarosław Bilińsk

jbilinski@wum.edu.p

Specialty section:

This article was submitted to

Hematologic Malignancies,

a section of the journal

Frontiers in Oncology

Received: 14 July 2021

Accepted: 13 September 2021

Published: 01 October 2021

Citation:

Jasiński M, Biliński J and Basak GW

(2021) The Role of the Gut Microbiome

in Pathogenesis, Biology, and

Treatment of Plasma Cell Dyscrasias.

Front. Oncol. 11:741376.

doi: 10.3389/fonc.2021.741376

\section{The Role of the Gut Microbiome in Pathogenesis, Biology, and Treatment of Plasma Cell Dyscrasias}

\author{
Marcin Jasiński ${ }^{1}$, Jarosław Biliński ${ }^{1,2 *}$ and Grzegorz W. Basak ${ }^{1,2}$ \\ ${ }^{1}$ Department of Hematology, Transplantation and Internal Medicine, Medical University of Warsaw, Warsaw, Poland, \\ 2 Human Biome Institute, Gdansk, Poland
}

In response to emerging discoveries, questions are mounting as to what factors are responsible for the progression of plasma cell dyscrasias and what determines responsiveness to treatment in individual patients. Recent findings have shown close interaction between the gut microbiota and multiple myeloma cells. For instance, that malignant cells shape the composition of the gut microbiota. We discuss the role of the gut microbiota in (i) the development and progression of plasma cell dyscrasias, and (ii) the response to treatment of multiple myeloma and highlight faecal microbiota transplantation as a procedure that could modify the risk of progression or sensitize refractory malignancy to immunotherapy.

Keywords: plasma cell dyscrasias, gut microbiome, multiple myeloma, microbiota, short-chain fatty acids

\section{INTRODUCTION - PATHOGENESIS OF PLASMA CELL DYSCRASIAS}

Typical genetic alterations in plasma cell dyscrasias are $\operatorname{IgH}$ translocations, hyperdiploidy, and cyclin D dysregulation. These are responsible for initiating changes in B-cell postgerminal centres, which result in the transformation of normal cells into benign tumour cells that cause monoclonal gammopathy of undetermined significance (MGUS) (1). This condition is the preclinical stage of multiple myeloma (MM) and occurs in $\sim 3.2 \%$ of the population aged over 50 years (2). MGUS is an asymptomatic condition with elevated serum concentration of $\mathrm{M}$ protein. Only rarely does it progress to symptomatic MM ( $1 \%$ of patients/year) (3), which can be associated with symptoms that manifest as a result of hypercalcaemia, renal failure, anaemia, and bone lesions. Smouldering MM (SMM) is an asymptomatic, intermediate stage between MGUS and MM, that carries a $10 \%$ risk of progression to symptomatic MM per year during the first five years after diagnosis (4). If it is to be possible to screen intensively, perform prophylactic investigations on, and treat in the early stages only those patients who are most at risk of disease progression, accurate prognostic markers of progression of MGUS or SMM to MM are needed.

During the past few years, evidence has emerged that human gut microbiota play an important role in the progression of MM (5-7). The gut microbiota influence the course of MM and the disease shapes the composition of the bacteria in the intestines (6). These interactions, as described below, are based on the strong reliance of MM cells on proinflammatory cytokines [interleukin (IL)-6, tumour necrosis factor (TNF)- $\alpha$, IL-13] and the ability of bacteria to recycle nitrogen (8). 
Recent studies have yielded plenty of information on the differences in microbiota among MM patients and about longitudinal changes acquired during the treatment as well (9). Some recently identified gut microbes are responsible for inducing an inflammatory environment, both within the gut layer and throughout the whole body. These proinflammatory microbes might contribute to the progression of MGUS to MM (5). If they do, the microbiome composition could be used as a prognostic factor for assessing the risk of MGUS transformation or MM progression.

\section{GUT MICROBIOTA AND IMMUNE SYSTEM IN HEALTH AND DISEASE, SPECIFICALLY INFECTIONS}

The colonization of the intestine by microbes plays a key role in the maturation of the host's immune system (10). Current knowledge about crosstalk between gut microbiota and immune cells derives mainly from experiments conducted on germ-free animals (11). For instance, in germ-free mice the population of $\alpha \beta$ and $\gamma \delta$ intraepithelial lymphocytes is significantly reduced (12), there is no production of IgA antibodies (13) and Th17 cells are absent (14). One example of a complicated interplay between gut microbiota and immune cells is the following. Polysaccharide A produced by Bacteroides fragilis binds to TLR2/TLR1 (Toll-like receptor) heterodimer connected with Dectin-1 (15). Then, the phosphoinositide 3-kinase (PI3K) pathway is activated, glycogen synthase kinase $3 \beta$ inactivated, which eventually induces cAMP response element-binding protein expression of anti-inflammatory genes (15). Finally, the secretion of polysaccharide A by Bacteroides fragilis leads to the differentiation of Treg cells and influences the balance between Th1 and Th2 populations. On the other hand, butyrate produced by the gut microbiota can promote macrophage differentiation from monocytes through histone deacetylase 3 (HDAC3) inhibition that leads to enhanced antimicrobial host defense (16). These are only a few examples of how intricate the crosstalk on the line gut microbiota - immune cells is.

Gut microbiota can also predict responses to therapies administered in oncology. Chaput et al. showed that the presence of Faecalibacterium spp. increases the efficacy of antiCTLA-4 immunotherapy while probably the Bacteroides spp. is associated with inferior responses in metastatic melanoma (17). Moreover, it is recently hypothesized that gut microbiota composition can influence the responses to the CAR-T therapy (18), and bearing in mind recent papers about the efficacy of such therapy in multiple myeloma the discussion about gut microbiota as a predictive marker of response is warranted (19).

The impact of the interplay between the immune system and gut microbiota in the context of infections cannot be forgotten as patients with multiple myeloma are far more prone to infections than the healthy population (20). The ability of microbes to release signaling molecules into the bloodstream can modulate the host's response to infections via the regulation of immune cell development (21). For instance, butyrate secreted by bacteria promotes the differentiation of monocytes in the bone marrow to a tolerogenic phenotype (22). Moreover, it was recently showed that some bacterial species could decrease the level of corticosterone in the blood which could improve the function of the immune system during the infection (23).

\section{GUT MICROBIOTA AND TUMOURIGENESIS}

The available data show that the gut microbiota are more numerous than genes, cells, and enzymatic reactions in the host organism, which suggest their importance for its health. In healthy persons, microorganisms are responsible for production of vitamins $\mathrm{K}, \mathrm{B}_{2}$ (riboflavin), $\mathrm{B}_{12}$ (cobalamin), folates, and biotin (24), metabolism of indigestible compounds, and protection from colonisation by opportunistic bacteria (25), and are necessary for the development of the humoral and cellular mucosal immune systems (26) (Figure 1). Along with these advantages of the gut microbiome, there are also some disadvantages. It is well established that dysbiosis, which is an imbalance in the proportion of microbes compared to a healthy state, plays a role in the pathogenesis of colorectal cancer (CRC) (27). Wang et al. showed that there is a difference in the composition of gut microbiota between patients with CRC and healthy individuals (28). A similar influence of microbial dysbiosis, via proinflammatory microbe-associated molecular patterns (MAMPs) and bacterial metabolites, has been shown in liver (29) and pancreatic (30) cancer.

The gut microbiota are accompanied by gut-associated lymphoid tissue (GALT), which is the largest peripheral immune organ (31). As many as $60-70 \%$ of peripheral lymphocytes are localised within the gut mucosa, so it is not surprising that the number of interactions between immune cells and the gut microbiota is high (32). There are numerous examples of how the gut microbiota and immune system influence each other within the gut mucosa. Brandsma et al. showed that the transplantation of proinflammatory faecal microbiota from $\mathrm{Casp1}^{-/-}$mice to $\mathrm{Ldlr}^{-1-}$ mice resulted in systemic inflammation and promoted atherogenesis (33). In contrast, Mason et al. reported that reduced anti-inflammatory gut microbiota was correlated positively with depression. This correlation could be explained by inflammation playing a role in the pathogenesis of depression (34). The crosstalk from microbes to immune cells can be forwarded directly through their metabolites used as messengers, such as MAMPs or damageassociated molecular patterns (DAMPs), or through activation of Toll-like receptors (TLRs) that in turn cause the activation of immune cells $(35,36)$. Some metabolites, such as short-chain fatty acids (SCFAs), can directly promote the generation of T regulatory (Treg) cells (37) or are responsible for transforming growth factor- $\beta$ production in epithelial cells within the gut. This in turn promotes Treg-cell confluence in the gut mucosa, which inhibits the activation of immune cells (38). Germ-free (GF) mice that are deprived completely of gut microbiota comprise excellent examples of the importance of gut bacteria for efficient immune function (26). In GF mice, Treg cell function is impaired, which suggests that gut microflora are necessary for the development of a fully functional 


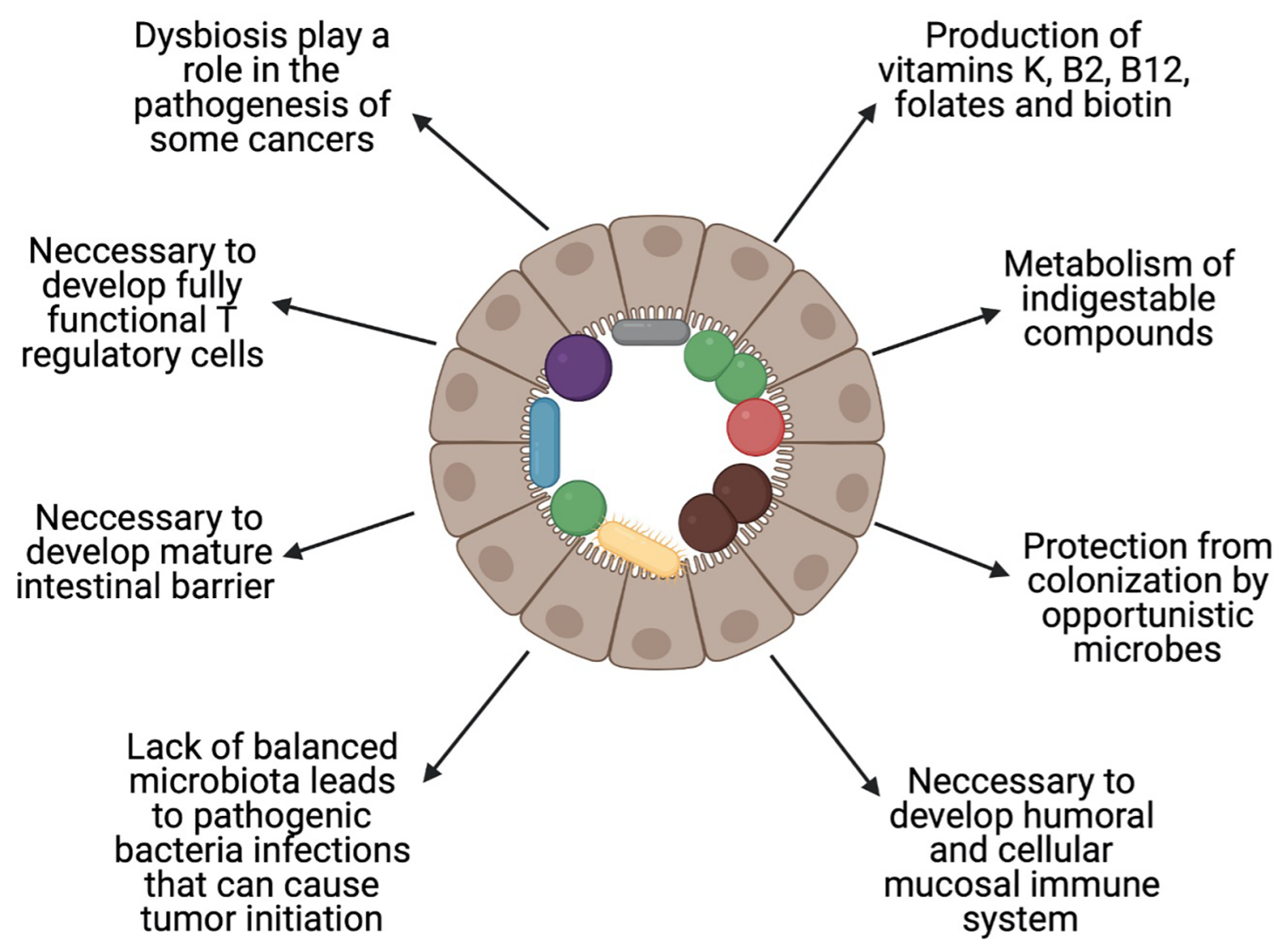

FIGURE 1 | Role of the balanced gut microbiota. Healthy gut microbiota are important in maintaining health. The figure shows the most important roles that are played by the human gut microbiota. Created with BioRender.com.

Treg cell population (39). In GF mice, the intestinal barrier is immature, which results in increased mucosal permeability (40). This is a key mechanism that leads to the development of inflammatory bowel disease or enteric infections (40). Colonisation of GF animals with normal gut microbiota leads to increased systemic immunological capacity, different patterns of migration of immune cells, significant changes in the production of specific antibodies, a general increase of immunoglobulin production, and changes in mucosal-associated lymphocyte tissues and cell populations (41-43).

In summary, in general, the micro-organisms in the gut are beneficial, but under certain conditions can have a damaging effect, in severe cases promoting the growth of cancer cells.

\section{COMPARISON OF THE GUT MICROBIOME IN PATIENTS WITH PLASMA CELL DYSCRASIAS AND HEALTHY INDIVIDUALS}

In recent years, scientists have confirmed the link between certain kinds of tumours and the composition of gut microbiota. For example, in CRC, many changes in the composition of bacterial species that colonise the gut have been identified and their contribution to tumourigenesis confirmed. Specific bacterial species colonizing the gut have even been indicated as possible markers of early diagnosis of CRC (44).

Regarding plasma cell dyscrasias and the gut microbiome, recent evidence shows metagenomic changes in the composition of commensal bacteria and frequent colonisation by opportunistic bacteria. Jian et al. performed a study on samples collected from 19 patients who had been newly diagnosed with MM and 18 healthy controls (6). They observed significant differences in the composition of bacteria in the gut between these two groups. One of the main changes was the increase of nitrogen-recycling bacteria, such as Klebsiella and Streptococcus, which are opportunistic pathogens that are responsible for infections associated with high mortality in this immunocompromised population. It has been suggested that this change might be due to the high serum concentration of urea in patients with $\mathrm{MM}$, which results from increased production of $\mathrm{NH}_{4}{ }^{+}$by tumour cells and restricted secretion of urea due to impaired renal function (45). The mechanism presented above is responsible attracting nitrogenrecycling bacteria to the gut. Changes in diversity in gut 
microbiota have been reported, which indicates that samples from MM patients are characterised by increased diversity and poorer interactions between genera (6), although other studies have produced results that indicate contrary phenomena $(46,47)$. Furthermore, samples from MM patients included a reduced number of SCFA-producing bacteria, which affect tumourigenesis in plasma cell dyscrasias (see below) (6). Other changes in the composition of commensal bacteria, and colonisation with opportunistic pathogens, occur because of the treatment of MM. Unfortunately, research in this field is limited to the study of bacterial composition only. Further research, which studies differences in the balance and numbers, etc., of fungi, viruses, and eukaryotic organisms are needed (Table 1).

\section{INFLUENCE OF THE GUT MICROBIOME ON THE DEVELOPMENT AND PROGRESSION OF PLASMA CELL DYSCRASIAS}

As mentioned previously, MGUS is an asymptomatic state that occurs in $\sim 3.2 \%$ of people aged over 50 (1). Only a small percentage of patients progress to symptomatic MM. For many years, researchers have wanted to identify the factors responsible for the development of plasma cell dyscrasias, and the reasons why some patients progress to MM whereas others do not.

Researchers have shown that there are no significant genetic differences between MGUS and MM cells. This suggests that environmental conditions could be an important factor in determining the risk for progression, although such factors are not necessarily present at the time at which MGUS develops. Therefore, tumour microenvironment seems to be a strong predictor of MGUS progression. Given the high degree of heterogeneity between clones in plasma cell dyscrasias, it is probable that only clones that are developing in a favourable niche will become an initiation point for further progression. As mentioned previously, proinflammatory TME in the bone marrow is needed for successful progression from MGUS to symptomatic MM, but it is a further issue how the gut microbiota can influence this microenvironment and contribute to tumour progression.

\section{Short-Chain Fatty Acids}

SCFAs are bacterial products that are responsible for ion absorption, gut motility, and modulation of immune responses (48). SCFAs can inhibit the nuclear factor kappa-light-chain

TABLE 1 | Summary of the alterations of the gut microbiota in MM patients.

Gut microbiota of MM patients

Frequently colonised with opportunistic bacteria (6)

Increase in the number of bacteria involved in nitrogen recycling, such as $K$.

pneumoniae or S, pneumoniae (6)

Increased diversity and poorer interactions between genera (6)

Reduced number of SCFA-producing bacteria (6)

Changes resulting from applied treatments especially antibiotics enhancer of activated B cells (NF- $\kappa \mathrm{B}$ ) and such proinflammatory cytokines as IL- 6 and TNF- $\alpha$ which are playing the role in activating osteoclasts to create niches for myeloma cells and additionally promote differentiation of Th17 cell (49). In contrast, SCFAs may also increase the level of IL-10 and induce expression of FoxP3 which in turn leads to differentiation of immunosuppressive $\mathrm{CD}^{+} \mathrm{T}$ cell subset (Treg) (48). Eventually, both Treg (IL-10 and TGF- $\beta$ ) and Th17 (IL-17) cells secrete cytokines that promote MM cell proliferation via positive feedback loop (50). One SCFA, butyrate, is reported to increase T-cell apoptosis by HDACdependent Fas upregulation and consequent Fas-mediated apoptosis of $\mathrm{T}$ cells. That in turn inhibit T-cell accumulation within inflamed colonic mucosa which could prevent antigenic stimulation known for its role in multiple myeloma development (51). Furthermore, Jian et al. showed that SCFA-producing bacteria such as Anaerostipes hadrus, Clostridium butyricum, and Clostridium saccharobutylicum were reduced in patients with MM, and that the addition of Clostridium butyricum in a mouse model of MM resulted in mitigation of tumour progression (6). SCFAs are also involved in the response to treatment. Small, uncontrolled studies have indicated that SCFAproducing bacteria play a significant role in reducing the level of proinflammatory cytokines, thereby protecting the host from tumour progression. Loss of SCFA-producing bacteria can result in a higher risk of tumour progression. Bearing in mind that specific diets can increase the population of SCFA-producing bacteria, studies are needed to investigate whether changes in diet in patients with MGUS can influence the risk of tumour progression.

\section{L-Glutamine}

Jian et al. showed that stool samples from MM patients had higher concentrations than in healthy patients of bacteria that are involved in nitrogen utilisation and recycling, such as Klebsiella and Streptococcus (6). The following mechanism has been proposed to explain this phenomenon (6). MM cells are known producers of $\mathrm{NH}_{4}{ }^{+}$(52), which results from uptake of glutamine (53). This $\mathrm{NH}_{4}{ }^{+}$then accumulates in the bone marrow and is released into the blood. In a healthy organism, the liver successfully converts $\mathrm{NH}_{4}{ }^{+}$into urea in the urea cycle. However, MM patients experience a high increase in blood $\mathrm{NH}_{4}{ }^{+}$level that exceeds the capacity of the liver to convert it to urea and can even result occasionally in hyperammonaemic encephalopathy (54). In addition, monoclonal protein renal deposition and consequent reduction in renal function mean that the process of urea excretion is impaired severely (55). Taken together, these factors lead to an increased concentration of urea in the blood, such that excessive amounts of urea reach the intestinal lumen. The presence of urea in the gut layer causes the selection of nitrogen-recycling bacteria, such as Klebsiella and Streptococcus. These bacteria are involved in the hydrolysis of urea and synthesis of L-glutamine that is taken up by MM cells, which promotes tumour progression. It is probable that MM cells harness the gut microbiota of the host as a recycler of $\mathrm{NH}_{4}^{+}$to deliver the necessary L-glutamine. In light of this, we speculate that targeting human microbiota with natural methods, or 
antibiotics, if necessary, could be an attractive strategy to stop this vicious cycle.

\section{Th17 Cells}

The differentiation of Th17 in GF mice is inhibited (14). Microbial colonization, especially with segmented filamentous bacteria (SFB) promotes induction of Th17 cells (56). Furthermore, it is already known that Th17 elicited by SFB are of non-inflammatory phenotype while Th17 cells induced by other bacteria Citrobacter are secreting plenty of proinflammatory cytokines (57).

Plasma cells express IL-17 receptors on their surface and are stimulated in vitro and in vivo via IL-17 produced by Th17 cells (58). Of note, IL-6-STAT3 signalling pathway activated by IL-17 is relevant both for tumour (59) and plasma cell (60) growth which suggests the role of IL-17 during different stages of MM. IL-17 causes the upregulation of the receptor activator of the NF$\kappa \mathrm{B}$ ligand, which results in the activation of osteoclasts (61) and eosinophils that are producing IL- 6 and TNF- $\alpha$ (5). Hence, IL-17 is the cytokine that bears the principal responsibility for bone lesions in plasma cell dyscrasias. Stromal cells respond to IL-17 as well by producing IL-6 (62). Moreover, the interplay between IL- 6 and TGF- $\beta$, that are highly expressed in the bone marrow of patients with MM, is influencing the generation of Th17 cells (49).

Prevotella heparinolytica is responsible for the differentiation of Th17 cells and their migration to the bone marrow in the $\mathrm{Vk}^{\star} \mathrm{MYC}$ mouse model of MM (5). In mice that lacked IL-17, the progression of plasma cell dyscrasias was delayed. Inhibition of IL-17, IL-17 receptor A, and IL-5 in a $\mathrm{Vk}^{\star} \mathrm{MYC}$ model with monoclonal antibodies results in reduced accumulation of Th17 cells and eosinophils in the bone marrow, which results in delayed tumour progression (5).

Patients with MM have elevated serum level of IL-17 but interestingly after therapy with bis-phosphonate level of that cytokine is reduced (63). A higher level of IL-17 is also seen in the blood of patients with SMM and is a predictor of rapid progression of tumour growth. Therefore the level of IL-17 could be used as a potential marker of high-risk SMM patients (64). Similar to the Vk MYC model, it would be useful to initiate studies on patients to determine which bacteria are involved in Th17 differentiation. Using this approach, bacteria that are involved indirectly in the development of bone lytic lesions, which is one of the main causes of morbidity in MM patients, could be eradicated (Figure 2).

\section{THE LINK BETWEEN THE GUT MICROBIOME AND TREATMENT IN PLASMA CELL DYSCRASIAS}

It is known that different results of treatment and toxicity profiles are associated with the gut microbiome $(65,66)$. For instance, a specific composition of gut microbiota is required for an optimal response to treatment with immune checkpoint inhibitors (67). Baruch et al. conducted a phase I study on faecal microbiota transplantation from complete responders to treatment for metastatic melanoma to 10 non-responders, which resulted in

GUT MICROBIOTA IN MM
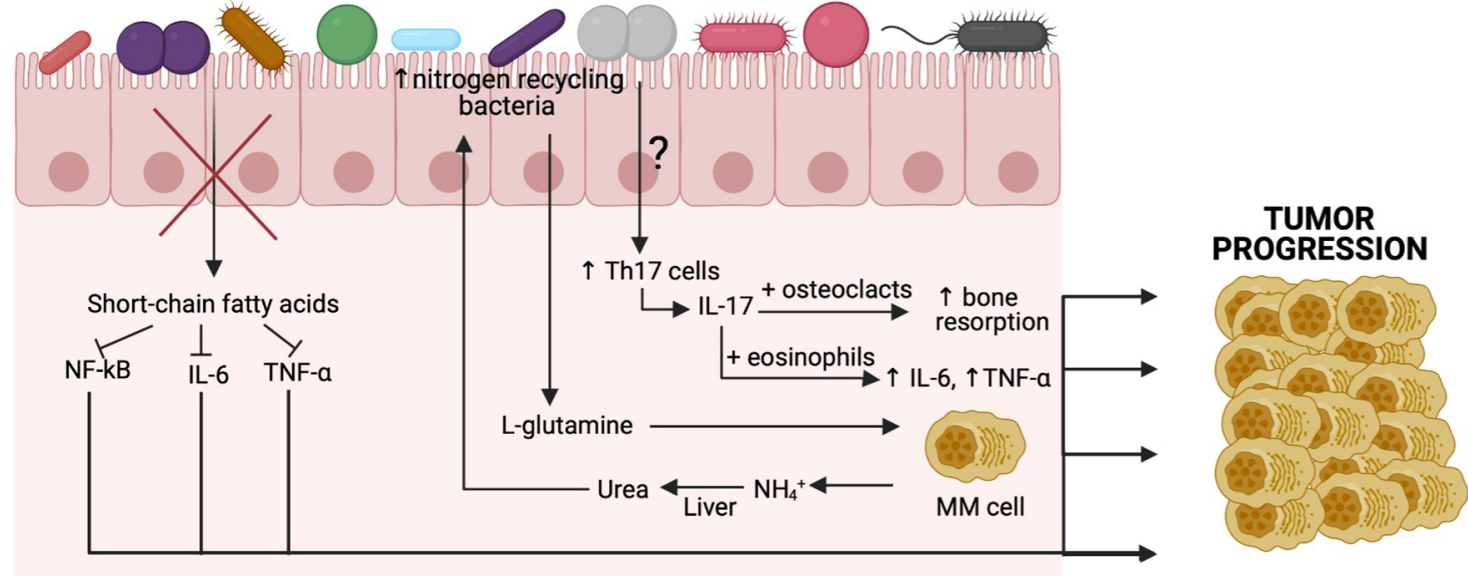

FIGURE 2 | Association between the gut microbiota and tumour progression in MM patients. Recent findings show a close relationship between gut commensal microbiota and MM cells. SCFA-producing bacteria are significantly reduced, resulting in increased levels of NF-kB, IL-6, and TNF- $\alpha$, which are known to contribute to tumor progression in MM. Another example derives from the fact that MM patients have increased nitrogen-recycling bacteria. These bacteria are involved in L-glutamine production, which is an essential amino acid for MM cells. MM cells produce high amounts of $\mathrm{NH}^{+}$, which is transformed in the liver into urea and reaches high concentrations in the blood and can select nitrogen-recycling bacteria such as K. pneumoniae or S. pneumoniae. The influence of the gut microbiota on Th17 cell differentiation in MM patients remains to be characterised, although we know that in a Vk*MYC mouse model, P. heparinolytica was responsible for that. Patients with MM have significantly higher level of IL-17 in the blood, which is produced by Th17 cells and causes bone resorption, resulting in bone lesions that are the main symptom of this malignancy. Additionally, IL-17 activates eosinophils that are consequently producing proinflammatory cytokines (IL-6 and TNF- $\alpha$ ) that are involved in tumor progression. Created with BioRender.com. 
partial responses in three patients and a complete response in one (68). The gut microbiome can influence the results of treatment, especially in respect of adverse events, and treatment can modulate the gut microbiome.

During the last decade, new treatments for plasma cell dyscrasias have been introduced, including immunomodulatory drugs (thalidomide, lenalidomide, and pomalidomide), proteasome inhibitors, and monoclonal antibodies. These have improved the length and quality of life of patients with MM (69). To emphasise the role of the gut microbiome in plasma cell dyscrasias, we describe how microbes can affect the outcomes of treatment in plasma cell malignancies. Their role is particularly visible in respect of possible infectious complications after treatment that are due to infection. It was recently confirmed that treatment of MM changes the composition of the gut microbiome in respect of diversity (70).

Pianko et al. showed that MM patients with no minimal residual disease (MRD) after completion of upfront therapy had greater numbers of butyrate-producing Eubacterium halii than MRD-positive patients (71). Similarly, another butyrate producer, Faecalibacterium prausnitzii, was associated with an absence of MRD (71). Moreover, Peled et al. showed that intestinal Eubacterium limosum was associated with decreased risk of MM relapse after allogeneic haematopoietic cell transplantation (72). These observations suggest that changes in commensal microbiota caused by MM treatment could influence the entire process of therapy or be a predictor of a better response. Gopalakrishnan et al. showed how significant the impact of the changes in the gut microflora on the response to treatment can be. They showed that melanoma patients who responded well to immunotherapy with anti-PD-1 agents had a relative abundance of Ruminococcaceae family and higher alpha diversity (diversity within one sample) in faecal microbiome samples (73). Thus, it is possible that the composition of gut microbiota in MM patients has a major influence on the outcomes of immunotherapy, especially taking into account that MM, similarly to melanoma, is closely related to immune response.

\section{Proteasome Inhibitors}

PIs, such as bortezomib or carfilzomib are commonly used in primary and relapsed MM. One common adverse effect is gastrointestinal (GI) toxicity that results in diarrhoea. First, it was thought that PIs alter gut motility or cause neurotoxicity, resulting in autonomic neuropathy. The molecular reason for GI toxicity is now established as an increase in TNF- $\alpha$ receptor 1 expression on intestinal cells and higher concentrations of IL-6, TNF- $\alpha$ and IL-1 $\beta$ (74). However, there is a lack of evidence that PIs influence composition of the gut microbiota. It might be that inhibition of the NF- $\kappa$ B pathway is responsible for GI toxicity of PIs (75). SCFAs can suppress the NF- $\kappa B$ pathway, which could augment GI toxicity of PIs (76).

\section{Steroids}

Steroids are among the most commonly used anti-inflammatory drugs. They are used in chemotherapy regimens for MM, as well as in the treatment of a wide range of rheumatoid diseases.
Huang et al. showed that mice that had been subjected to chronic exposure to steroids differed in the composition of their gut microbiota compared with their healthy counterparts (77). Steroid-treated mice had an increase in Bifidobacterium and Lactobacillus, which are both associated with anti-inflammatory effects, whereas they noted an absence of Mucospirillum, which is responsible for degradation of colonic mucin. This effect might be explained by the decrease of mucin production in mice treated chronically with steroids. Dexamethasone exerts its antiinflammatory effects by blocking the NF- $\mathrm{KB}$ pathway (78). Furthermore, mice that were treated with dexamethasone produced less IL-17 than healthy mice (77). This may be another case in which steroids reshape the intestinal flora, since IL-17 production depends on Th17 cell differentiation, which is associated with specific gut microbiota. However, not only chronic exposure to, but also acute treatment with, steroids affected gut microbiota in mice (77). Ünsal et al. showed that rodents that were injected with a single, strong dose of dexamethasone underwent an increase in the number of ileal anaerobic bacteria. Moreover, a single injection of a low dose of dexamethasone resulted in an increase in the population of coliform bacteria (79). However, the long-term effect of these changes remains to be determined.

\section{Antimicrobials}

The link between antibiotics and the gut microbiome seems to be the most examined and the influence of this group of drugs on commensal bacteria is well established. However, although this link has been studied intensively in healthy volunteers, there remains a lack of wider studies with many groups of antibiotics in MM patients. Ziegler et al. showed that levofloxacin, which is the most commonly prescribed drug for bloodstream infections and neutropenic fever prophylaxis, had a less damaging effect on intestinal microbiota than broad-spectrum $\beta$-lactam (BSBL) antibiotics (80). The latter group reduced alpha diversity. The former was not associated with specific changes in the gut microbiome that had been found to be associated with poor clinical results (decrease in populations responsible for protection against $C$. difficile; increase in non-Bacteroidetes taxa, and reduction of alpha diversity). In light of their results, the authors emphasised that fluoroquinolone antibiotics protected patients from the negative effects of BSBLs (80). In MM patients who had been newly diagnosed and who were at particular risk of infection, the effect of prophylactic antibiotics was small and there was no decrease in early mortality (81). However, Valkovic et al. reported that MM diagnosis or progression was frequently preceded by infection (82). That could have been because bacterial infections are associated with robust production of proinflammatory cytokines and TLR activation on MM cells $(83,84)$. This is why prophylactic broadspectrum antibiotics can result in a delay in disease progression. In respect of allogeneic stem-cell transplantation (alloSCT), Weber et al. showed that early use of broad-spectrum antibiotics that are active against commensal organisms, such as Clostridiales was associated with increased transplant-related mortality and decreased overall survival (85). Administration of imipenem-cilastatin or piperacillin-tazobactam for neutropenic 
fever resulted in gut microbial perturbation and increased graftversus-host disease-related mortality compared with aztreonam or cefepime, both of which decreased activity against commensal, anaerobic bacteria (86). Such observations of antibiotic effects on the response to treatment of $\mathrm{MM}$ need to be investigated in patients who are treated with autologous stem-cell transplantation (ASCT). There is also a recently published systematic review of infections associated with selinexor in patients with relapsed/refractory MM that also compares the risk of infections with other novel agents. It is already known that selinexor could prevent viral infections through blocking of XPO1 - mediated nuclear transport which facilitates the export of viral proteins. The authors state that randomized clinical trials are needed to fully understand the risk of infections associated with selinexor (87).

\section{Autologous Stem-Cell Transplantation}

D'Angelo showed that after ASCT, patients showed significantly decreased diversity of the microbial gut population (88). El Jurdi et al. showed an association between baseline microbiota of patients undergoing ASCT with further regimen-related toxicities and with the rate of neutrophil engraftment (89). They found that bacterial diversity after ASCT recovered within 1 month after the procedure, but that fungal populations constantly decreased, which suggests that a longer time is needed for the reconstitution of the mycobiome. Although the prospective study included only 15 patients, the results were encouraging for further studies. This group recognised several links between the composition of the microbiota and effects on ASCT-related toxicity and outcomes. One of the links relied on identifying an increased population of Bacteroides at day +7 in patients with less severe diarrhoea, while more severe diarrhoea, nausea, and vomiting occurred in patients with a higher prevalence of the stool populations of Blautia and Ruminococcus. They also identified a negative correlation between fungal phyla Glomerella presence in stools and neutrophil engraftment (89). Similar conclusions were drawn from the results of the small pilot study with 15 patients, showing that baseline microbiota were associated with subsequent incidence and severity of nausea, vomiting, neutropenic fever, and rate of neutrophil engraftment (90). Khan et al. showed recently that 534 adult recipients of highdose chemotherapy with ASCT had significantly decreased alpha diversity at early pretransplant stages than healthy individuals and that this reduction in diversity tended to be more marked in the course of the procedure (9). The pattern of this loss of diversity and dominance of specific taxa were similar to those seen in patients after alloSCT. In addition, they showed that the greater the diversity of the gut microbiota, the lower risk of progression or death. Our group showed in a retrospective, single-centre study that colonisation with antibiotic-resistant bacteria had a significant influence on the outcomes of alloSCT (91). The main finding was that the overall survival of patients who were colonised by antibiotic-resistant bacteria was estimated to be half that of the noncolonised group. A similar conclusion was reached by Scheich et al. concerning the effect of colonisation by multidrug-resistant organisms on the results of ASCT (92).

\section{Other Treatments}

There is little information on the possible influence of other treatments, such as immunomodulatory drugs and monoclonal antibodies, on plasma cell dyscrasias (Table 2).

\section{CONCLUSIONS}

Despite some progress in the outcomes of treatment of MM, it remains a disease that cannot currently be cured, due to relapse or refractoriness to any available therapy. An emerging factor that could influence not only the refractoriness of MM but also a progression from asymptomatic MGUS to MM is the gut microbiota. We see that changes in the composition of

TABLE 2 | Relationship between the gut microbiota and treatment of plasma cell dyscrasias.

\section{Treatment}

Pls - There is no evidence proving the influence of Pls on gut microbiota

Steroids - Mice treated with steroids had increased Bifidobacterium and Lactobacillus population and the absence of Mucospirillum bacteria (77)

- $\quad$ Mice treated with dexamethasone had decreased production of IL-17 compared with an untreated group. IL-17 production is strictly related to the presence of Th17 cells, whose differentiation in the gut was recently proved in the Vk* MYC mouse model. This indicates some relationship (77)

- $\quad$ Not only chronic exposure but also acute treatment resulted in alteration of the gut microbiota in rodents (79)

Antimicrobials - Levofloxacin had no significant impact on the human gut microbiota, while BSBL antibiotics caused a reduction of alpha diversity (80)

- Administration of broad-spectrum antibiotics efficient against commensal microbiota resulted in higher transplant-related mortality and decreased overall survival (85)

- $\quad$ Patients treated with imipenem-cilastatin or piperacillin-tazobactam had increased risk of GVHD-related mortality compared with aztreonam or cefepime (86)

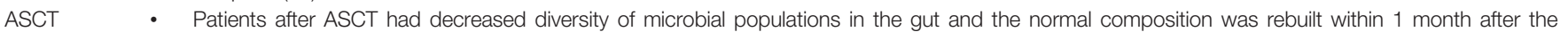
procedure (89)

- $\quad$ There is a strong relationship between baseline microbiota of MM patients and severity of toxicity related to the procedure and with the rate of neutrophil engraftment (89)

- $\quad$ Patients after high-dose chemotherapy before ASCT had significantly decreased alpha diversity of the gut microbiota compared with healthy individuals (9)

Other - Little is known about possible influence of gut microbiome on treatment outcomes with immunomodulatory drugs or monoclonal antibodies 
commensal bacteria can affect the process of transforming MGUS to MM. Further, these changes are associated with colonisation with opportunistic pathogens that can become an aetiological agent of complications due to infection that are associated with treatment. Probably, in the future, it will be possible to identify patients who have an especially high risk of progression to $\mathrm{MM}$, or even to modulate intestinal microflora to reduce the risk of progression of MGUS. It is also possible that the gut microbiota will be modulated to reduce complications that are due to treatment and disease, or to

\section{REFERENCES}

1. Agarwal A, Ghobrial IM. Monoclonal Gammopathy of Undetermined Significance and Smoldering Multiple Myeloma: A Review of the Current Understanding of Epidemiology, Biology, Risk Stratification, and Management of Myeloma Precursor Disease. Clin Cancer Res (2013) 19 (5):985-94. doi: 10.1158/1078-0432.CCR-12-2922

2. Kyle RA, Therneau TM, Rajkumar SV, Larson DR, Plevak MF, Offord JR, et al. Prevalence of Monoclonal Gammopathy of Undetermined Significance. N Engl J Med (2006) 354(13):1362-9. doi: 10.1056/NEJMoa054494

3. Kyle RA, Therneau TM, Rajkumar SV, Offord JR, Larson DR, Plevak MF, et al. A Long-Term Study of Prognosis in Monoclonal Gammopathy of Undetermined Significance. N Engl J Med (2002) 346(8):564-9. doi: 10.1056/ NEJMoa01133202

4. Kyle RA, Remstein ED, Therneau TM, Dispenzieri A, Kurtin PJ, Hodnefield JM, et al. Clinical Course and Prognosis of Smoldering (Asymptomatic) Multiple Myeloma. N Engl J Med (2007) 356(25):2582-90. doi: 10.1056/ NEJMoa070389

5. Calcinotto A, Brevi A, Chesi M, Ferrarese R, Garcia Perez L, Grioni M, et al. Microbiota-Driven Interleukin-17-Producing Cells and Eosinophils Synergize to Accelerate Multiple Myeloma Progression. Nat Commun (2018) 9(1):4832. doi: 10.1038/s41467-018-07305-8

6. Jian X, Zhu Y, Ouyang J, Wang Y, Lei Q, Xia J, et al. Alterations of Gut Microbiome Accelerate Multiple Myeloma Progression by Increasing the Relative Abundances of Nitrogen-Recycling Bacteria. Microbiome (2020) 8 (1):74. doi: 10.1186/s40168-020-00854-5

7. D'Angelo CR, Sudakaran S, Callander NS. Clinical Effects and Applications of the Gut Microbiome in Hematologic Malignancies. Cancer (2021) 127 (5):679-87. doi: 10.1002/cncr.33400

8. Ahmed N, Ghannoum M, Gallogly M, de Lima M, Malek E. Influence of Gut Microbiome on Multiple Myeloma: Friend or Foe? J Immunother Cancer (2020) 8(1):1-3. doi: 10.1136/jitc-2020-000576

9. Khan N, Lindner S, Gomes ALC, Devlin SM, Shah G, Sung AD, et al. Fecal Microbiota Diversity Disruption and Clinical Outcomes After Auto-HCT: A Multicenter Observational Study. Blood (2020) 137:13-5. doi: 10.1182/ blood.2020006923

10. Gensollen T, Iyer SS, Kasper DL, Blumberg RS. How Colonization by Microbiota in Early Life Shapes the Immune System. Science (2016) 352 (6285):539-44. doi: 10.1126/science.aad9378

11. Bauer H, Horowitz RE, Levenson SM, Popper H. The Response of the Lymphatic Tissue to the Microbial Flora. Studies on Germfree Mice. Am J Pathol (1963) 42:471-83.

12. Umesaki Y, Setoyama H, Matsumoto S, Okada Y . Expansion of Alpha Beta TCell Receptor-Bearing Intestinal Intraepithelial Lymphocytes After Microbial Colonization in Germ-Free Mice and its Independence From Thymus. Immunology (1993) 79(1):32-7.

13. Hapfelmeier S, Lawson MA, Slack E, Kirundi JK, Stoel M, Heikenwalder M, et al. Reversible Microbial Colonization of Germ-Free Mice Reveals the Dynamics of Iga Immune Responses. Science (2010) 328(5986):1705-9. doi: $10.1126 /$ science. 1188454

14. Ivanov II, Frutos Rde L, Manel N, Yoshinaga K, Rifkin DB, Sartor RB, et al. Specific Microbiota Direct the Differentiation of IL-17-Producing T-Helper Cells in the Mucosa of the Small Intestine. Cell Host Microbe (2008) 4(4):33749. doi: 10.1016/j.chom.2008.09.009 improve treatment outcomes. However, the field of microbiota in MM is still in its infancy and further work is required to gain a fuller understanding of the phenomena.

\section{AUTHOR CONTRIBUTIONS}

All authors contributed to the article and approved the submitted version.

15. Erturk-Hasdemir D, Oh SF, Okan NA, Stefanetti G, Gazzaniga FS, Seeberger $\mathrm{PH}$, et al. Symbionts Exploit Complex Signaling to Educate the Immune System. Proc Natl Acad Sci USA (2019) 116:26157-66. doi: 10.2139/ ssrn. 3362573

16. Schulthess J, Pandey S, Capitani M, Rue-Albrecht KC, Arnold I, Franchini F, et al. The Short Chain Fatty Acid Butyrate Imprints an Antimicrobial Program in Macrophages. Immunity (2019) 50(2):432-445 e7. doi: 10.1016/ j.immuni.2018.12.018

17. Chaput N, Lepage P, Coutzac C, Soularue E, Le Roux K, Monot C, et al. Baseline Gut Microbiota Predicts Clinical Response and Colitis in Metastatic Melanoma Patients Treated With Ipilimumab. Ann Oncol (2017) 28(6):136879. doi: 10.1093/annonc/mdx108

18. Abid MB, Shah NN, Maatman TC, Hari PN. Gut Microbiome and CAR-T Therapy. Exp Hematol Oncol (2019) 8:31. doi: 10.1186/s40164-019-0155-8

19. Jasinski M, Basak GW, Jedrzejczak WW. Perspectives for the Use of CAR-T Cells for the Treatment of Multiple Myeloma. Front Immunol (2021) 12:632937. doi: 10.3389/fimmu.2021.632937

20. Blimark C, Holmberg E, Mellqvist UH, Landgren O, Bjorkholm M, Hultcrantz M, et al. Multiple Myeloma and Infections: A Population-Based Study on 9253 Multiple Myeloma Patients. Haematologica (2015) 100(1):107-13. doi: 10.3324/haematol.2014.107714

21. Khosravi A, Yanez A, Price JG, Chow A, Merad M, Goodridge HS, et al. Gut Microbiota Promote Hematopoiesis to Control Bacterial Infection. Cell Host Microbe (2014) 15(3):374-81. doi: 10.1016/j.chom.2014.02.006

22. Gao Y, Zhou J, Qi H, Wei J, Yang Y, Yue J, et al. Lncrna Lncly6c Induced by Microbiota Metabolite Butyrate Promotes Differentiation of Ly6C(High) to Ly6C(Int/Neg) Macrophages Through Lncly6c/C/Ebpbeta/Nr4A1 Axis. Cell Discov (2020) 6(1):87. doi: 10.1038/s41421-020-00211-8

23. Menezes-Garcia Z, Do Nascimento Arifa RD, Acurcio L, Brito CB, Gouvea JO, Lima RL, et al. Colonization by Enterobacteriaceae is Crucial for Acute Inflammatory Responses in Murine Small Intestine via Regulation of Corticosterone Production. Gut Microbes (2020) 11(6):1531-46. doi: 10.1080/19490976.2020.1765946

24. Hill MJ. Intestinal Flora and Endogenous Vitamin Synthesis. Eur J Cancer Prev (1997) 6(Suppl 1):S43-5. doi: 10.1097/00008469-199703001-00009

25. Round JL, Mazmanian SK. The Gut Microbiota Shapes Intestinal Immune Responses During Health and Disease. Nat Rev Immunol (2009) 9(5):313-23. doi: $10.1038 /$ nri2515

26. Cebra JJ. Influences of Microbiota on Intestinal Immune System Development. Am J Clin Nutr (1999) 69(5):1046S-51S. doi: 10.1093/ajcn/ 69.5.1046s

27. Zackular JP, Baxter NT, Iverson KD, Sadler WD, Petrosino JF, Chen GY, et al. The Gut Microbiome Modulates Colon Tumorigenesis. mBio (2013) 4(6): e00692-13. doi: 10.1128/mBio.00692-13

28. Wang T, Cai G, Qiu Y, Fei N, Zhang M, Pang X, et al. Structural Segregation of Gut Microbiota Between Colorectal Cancer Patients and Healthy Volunteers. ISME J (2012) 6(2):320-9. doi: 10.1038/ismej.2011.109

29. Dapito DH, Mencin A, Gwak GY, Pradere JP, Jang MK, Mederacke I, et al. Promotion of Hepatocellular Carcinoma by the Intestinal Microbiota and TLR4. Cancer Cell (2012) 21(4):504-16. doi: 10.1016/j.ccr.2012.02.007

30. Ochi A, Nguyen AH, Bedrosian AS, Mushlin HM, Zarbakhsh S, Barilla R, et al. Myd88 Inhibition Amplifies Dendritic Cell Capacity to Promote Pancreatic Carcinogenesis via Th2 Cells. J Exp Med (2012) 209(9):1671-87. doi: 10.1084/ jem.20111706 
31. Ohno H. Intestinal M Cells. J Biochem (2016) 159(2):151-60. doi: 10.1093/jb/ mvv121

32. Macpherson AJ, McCoy KD, Johansen FE, Brandtzaeg P. The Immune Geography of Iga Induction and Function. Mucosal Immunol (2008) 1 (1):11-22. doi: 10.1038/mi.2007.6

33. Brandsma E, Kloosterhuis NJ, Koster M, Dekker DC, Gijbels MJJ, van der Velden S, et al. A Proinflammatory Gut Microbiota Increases Systemic Inflammation and Accelerates Atherosclerosis. Circ Res (2019) 124(1):94100. doi: 10.1161/CIRCRESAHA.118.313234

34. Mason BL, Li Q, Minhajuddin A, Czysz AH, Coughlin LA, Hussain SK, et al. Reduced Anti-Inflammatory Gut Microbiota are Associated With Depression and Anhedonia. J Affect Disord (2020) 266:394-401. doi: 10.1016/j.jad.2020. 01.137

35. Chassaing B, Ley RE, Gewirtz AT. Intestinal Epithelial Cell Toll-Like Receptor 5 Regulates the Intestinal Microbiota to Prevent Low-Grade Inflammation and Metabolic Syndrome in Mice. Gastroenterology (2014) 147(6):1363-77 e17. doi: 10.1053/j.gastro.2014.08.033

36. Sanchez JMS, Doty DJ, DePaula-Silva AB, Brown DG, Bell R, Klag KA, et al. Molecular Patterns From a Human Gut-Derived Lactobacillus Strain Suppress Pathogenic Infiltration of Leukocytes Into the Central Nervous System. J Neuroinflamm (2020) 17(1):291. doi: 10.1186/s12974-020-01959-2

37. Furusawa Y, Obata Y, Fukuda S, Endo TA, Nakato G, Takahashi D, et al. Commensal Microbe-Derived Butyrate Induces the Differentiation of Colonic Regulatory T Cells. Nature (2013) 504(7480):446-50. doi: 10.1038/ nature 12721

38. Martin-Gallausiaux C, Beguet-Crespel F, Marinelli L, Jamet A, Ledue F, Blottiere HM, et al. Butyrate Produced by Gut Commensal Bacteria Activates TGF-Betal Expression Through the Transcription Factor SP1 in Human Intestinal Epithelial Cells. Sci Rep (2018) 8(1):9742. doi: 10.1038/ s41598-018-28048-y

39. Ostman S, Rask C, Wold AE, Hultkrantz S, Telemo E. Impaired Regulatory T Cell Function in Germ-Free Mice. Eur J Immunol (2006) 36(9):2336-46. doi: 10.1002/eji.200535244

40. Ukena SN, Singh A, Dringenberg U, Engelhardt R, Seidler U, Hansen W, et al. Probiotic Escherichia Coli Nissle 1917 Inhibits Leaky Gut by Enhancing Mucosal Integrity. PloS One (2007) 2(12):e1308. doi: 10.1371/journal.pone.0001308

41. Stepankova R, Sinkora J, Hudcovic T, Kozakova H, Tlaskalova-Hogenova H. Differences in Development of Lymphocyte Subpopulations From GutAssociated Lymphatic Tissue (GALT) of Germfree and Conventional Rats: Effect of Aging. Folia Microbiol (Praha) (1998) 43(5):531-4. doi: 10.1007/ BF02820814

42. Tlaskalova-Hogenova H, Sterzl J, Stepankova R, Dlabac V, Veticka V, Rossmann P, et al. Development of Immunological Capacity Under Germfree and Conventional Conditions. Ann N Y Acad Sci (1983) 409:96113. doi: $10.1111 / j .1749-6632.1983 . t b 26862 . x$

43. Tlaskalova-Hogenova H, Cerna J, Mandel L. Peroral Immunization of Germfree Piglets: Appearance of Antibody-Forming Cells and Antibodies of Different Isotypes. Scand J Immunol (1981) 13(5):467-72. doi: 10.1111/j.13653083.1981.tb00158.x

44. Dai Z, Coker OO, Nakatsu G, Wu WKK, Zhao L, Chen Z, et al. Multi-Cohort Analysis of Colorectal Cancer Metagenome Identified Altered Bacteria Across Populations and Universal Bacterial Markers. Microbiome (2018) 6(1):70. doi: 10.1186/s40168-018-0451-2

45. Howman R, Thakerer A, Pitman M, Ding N, Thompson PA, Khot A, et al. Bortezomib, Cyclophosphamide, and Dexamethasone: Highly Effective for Rapid Reversal of Myeloma-Associated Hyperammonemic Encephalopathy. Leuk Lymphoma (2010) 51(12):2299-302. doi: 10.3109/10428194.2010.518654

46. Antoine Pepeljugoski C, Morgan G, Braunstein M. Analysis of Intestinal Microbiome in Multiple Myeloma Reveals Progressive Dysbiosis Compared to MGUS and Healthy Individuals. Blood (2019) 134(Supplement_1):3076-6. doi: 10.1182/blood-2019-130643

47. Zhang B, Gu J, Liu J, Huang B, Li J. Fecal Microbiota Taxonomic Shifts in Chinese Multiple Myeloma Patients Analyzed by Quantitative Polimerase Chain Reaction (QPCR) and 16S Rrna High-Throughput Sequencing. Med Sci Monit (2019) 25:8269-80. doi: 10.12659/MSM.919988

48. Kim CH, Park J, Kim M. Gut Microbiota-Derived Short-Chain Fatty Acids, T Cells, and Inflammation. Immune Netw (2014) 14(6):277-88. doi: 10.4110/ in.2014.14.6.277
49. Hideshima T, Mitsiades C, Tonon G, Richardson PG, Anderson KC, et al. Understanding Multiple Myeloma Pathogenesis in the Bone Marrow to Identify New Therapeutic Targets. Nat Rev Cancer (2007) 7(8):585-98. doi: $10.1038 / \mathrm{nrc} 2189$

50. Manzo VE, Bhatt AS. The Human Microbiome in Hematopoiesis and Hematologic Disorders. Blood (2015) 126(3):311-8. doi: 10.1182/blood2015-04-574392

51. Zimmerman MA, Singh N, Martin PM, Thangaraju M, Ganapathy V, Waller $\mathrm{JL}$, et al. Butyrate Suppresses Colonic Inflammation Through HDAC1Dependent Fas Upregulation and Fas-Mediated Apoptosis of T Cells. Am J Physiol Gastrointest Liver Physiol (2012) 302(12):G1405-15. doi: 10.1152/ ajpgi.00543.2011

52. Otsuki T, Yamada O, Sakaguchi H, Ichiki T, Kouguchi K, Wada H, et al. In Vitro Excess Ammonia Production in Human Myeloma Cell Lines. Leukemia (1998) 12(7):1149-58. doi: 10.1038/sj.leu.2401077

53. Bolzoni M, Chiu M, Accardi F, Vescovini R, Airoldi I, Storti P, et al. Dependence on Glutamine Uptake and Glutamine Addiction Characterize Myeloma Cells: A New Attractive Target. Blood (2016) 128(5):667-79. doi: 10.1182/blood-2016-01-690743

54. Pham A, Reagan JL, Castillo JJ. Multiple Myeloma-Induced Hyperammonemic Encephalopathy: An Entity Associated With High in-Patient Mortality. Leuk Res (2013) 37(10):1229-32. doi: 10.1016/j.leukres.2013.07.014

55. Kourelis TV, Nasr SH, Dispenzieri A, Kumar SK, Gertz MA, Fervenza FC, et al. Outcomes of Patients With Renal Monoclonal Immunoglobulin Deposition Disease. Am J Hematol (2016) 91(11):1123-8. doi: 10.1002/ ajh. 24528

56. Ivanov II, Atarashi K, Manel N, Brodie EL, Shima T, Karaoz U, et al. Induction of Intestinal Th17 Cells by Segmented Filamentous Bacteria. Cell (2009) 139 (3):485-98. doi: 10.1016/j.cell.2009.09.033

57. Omenetti S, Bussi C, Metidji A, Iseppon A, Lee S, Tolaini M, et al. The Intestine Harbors Functionally Distinct Homeostatic Tissue-Resident and Inflammatory Th17 Cells. Immunity (2019) 51(1):77-89 e6. doi: 10.1016/ j.immuni.2019.05.004

58. Prabhala RH, Pelluru D, Fulciniti M, Prabhala HK, Nanjappa P, Song W, et al. Elevated IL-17 Produced by TH17 Cells Promotes Myeloma Cell Growth and Inhibits Immune Function in Multiple Myeloma. Blood (2010) 115(26):538592. doi: 10.1182/blood-2009-10-246660

59. Wang L, Yi T, Kortylewski M, Pardoll DM, Zeng D, Yu H. IL-17 can Promote Tumor Growth Through an IL-6-Stat3 Signaling Pathway. J Exp Med (2009) 206(7):1457-64. doi: 10.1084/jem.20090207

60. Palumbo A, Anderson K. Multiple Myeloma. N Engl J Med (2011) 364 (11):1046-60. doi: 10.1056/NEJMra1011442

61. Noonan K, Marchionni L, Anderson J, Pardoll D, Roodman GD, Borrello I. A Novel Role of IL-17-Producing Lymphocytes in Mediating Lytic Bone Disease in Multiple Myeloma. Blood (2010) 116(18):3554-63. doi: 10.1182/blood2010-05-283895

62. Prabhala RH, Fulciniti M, Pelluru D, Rashid N, Nigroiu A, Nanjappa P, et al. Targeting IL-17A in Multiple Myeloma: A Potential Novel Therapeutic Approach in Myeloma. Leukemia (2016) 30(2):379-89. doi: 10.1038/leu.2015.228

63. Oteri G, Allegra A, Bellomo G, Alonci A, Nastro E, Penna G, et al. Reduced Serum Levels of Interleukin 17 in Patients With Osteonecrosis of the Jaw and in Multiple Myeloma Subjects After Bisphosphonates Administration. Cytokine (2008) 43(2):103-4. doi: 10.1016/j.cyto.2008.05.010

64. Rajkumar SV, Landgren O, Mateos MV. Smoldering Multiple Myeloma. Blood (2015) 125(20):3069-75. doi: 10.1182/blood-2014-09-568899

65. Hooper LV, Littman DR, Macpherson AJ. Interactions Between the Microbiota and the Immune System. Science (2012) 336(6086):1268-73. doi: $10.1126 /$ science. 1223490

66. Ivanov II, Honda K. Intestinal Commensal Microbes as Immune Modulators. Cell Host Microbe (2012) 12(4):496-508. doi: 10.1016/j.chom.2012.09.009

67. Matson V, Fessler J, Bao R, Chongsuwat T, Zha Y, Alegre ML, et al. The Commensal Microbiome is Associated With Anti-PD-1 Efficacy in Metastatic Melanoma Patients. Science (2018) 359(6371):104-8. doi: 10.1126/ science.aao3290

68. Baruch EN, Youngster I, Ben-Betzalel G, Ortenberg R, Lahat A, Katz L, et al Fecal Microbiota Transplant Promotes Response in ImmunotherapyRefractory Melanoma Patients. Science (2021) 371(6529):602-9. doi: 10.1126/science.abb5920 
69. Naymagon L, Abdul-Hay M. Novel Agents in the Treatment of Multiple Myeloma: A Review About the Future. J Hematol Oncol (2016) 9(1):52. doi: 10.1186/s13045-016-0282-1

70. Ashraf A, Shaha N, Zahoor A, Arafat AF, Saif UR, Syeda SB, et al. Intestinal Microbiome Diversity and Clinical Outcomes With Multiple Myeloma: A Systematic Review. J Clin Oncol (2020) 38(15_suppl):e20542-2. doi: 10.1200/ JCO.2020.38.15_suppl.e20542

71. Pianko MJ, Devlin SM, Littmann ER, Chansakul A, Mastey D, Salcedo M, et al. Minimal Residual Disease Negativity in Multiple Myeloma is Associated With Intestinal Microbiota Composition. Blood Adv (2019) 3(13):2040-4. doi: 10.1182/bloodadvances. 2019032276

72. Peled JU, Devlin SM, Staffas A, Lumish M, Khanin R, Littmann ER, et al. Intestinal Microbiota and Relapse After Hematopoietic-Cell Transplantation. J Clin Oncol (2017) 35(15):1650-9. doi: 10.1200/JCO.2016.70.3348

73. Gopalakrishnan V, Spencer CN, Nezi L, Reuben A, Andrews MC, Karpinets TV, et al. Gut Microbiome Modulates Response to Anti-PD-1 Immunotherapy in Melanoma Patients. Science (2018) 359(6371):97-103. doi: $10.1126 /$ science.aan 4236

74. Stansborough RL, Gibson RJ. Proteasome Inhibitor-Induced Gastrointestinal Toxicity. Curr Opin Support Palliat Care (2017) 11(2):133-7. doi: 10.1097/ SPC. 0000000000000266

75. Al-Homsi AS, Feng Y, Duffner U, Al Malki MM, Goodyke A, Cole K, et al. Bortezomib for the Prevention and Treatment of Graft-Versus-Host Disease After Allogeneic Hematopoietic Stem Cell Transplantation. Exp Hematol (2016) 44(9):771-7. doi: 10.1016/j.exphem.2016.05.005

76. Alkharabsheh O, Sidiqi MH, Aljama MA, Gertz MA, Frankel AE. The Human Microbiota in Multiple Myeloma and Proteasome Inhibitors. Acta Haematol (2020) 143(2):118-23. doi: 10.1159/000500976

77. Huang EY, Inoue T, Leone VA, Dalal S, Touw K, Wang Y, et al. Using Corticosteroids to Reshape the Gut Microbiome: Implications for Inflammatory Bowel Diseases. Inflamm Bowel Dis (2015) 21(5):963-72. doi: 10.1097/MIB.0000000000000332

78. Scheinman RI, Gualberto A, Jewell CM, Cidlowski JA, Baldwin AS Jr. Characterization of Mechanisms Involved in Transrepression of NF-Kappa B by Activated Glucocorticoid Receptors. Mol Cell Biol (1995) 15(2):943-53. doi: 10.1128/MCB.15.2.943

79. Unsal H, Balkaya M, Unsal C, Biyik H, Basbulbul G, Poyrazoglu E. The ShortTerm Effects of Different Doses of Dexamethasone on the Numbers of Some Bacteria in the Ileum. Dig Dis Sci (2008) 53(7):1842-5. doi: 10.1007/s10620007-0089-6

80. Ziegler M, Han JH, Landsburg D, Pegues D, Reesey E, Gilmar C, et al. Impact of Levofloxacin for the Prophylaxis of Bloodstream Infection on the Gut Microbiome in Patients With Hematologic Malignancy. Open Forum Infect Dis (2019) 6(7):ofz252. doi: 10.1093/ofid/ofz252

81. Mohyuddin GR, Aziz M, McClune B, Abdallah AO, Qazilbash M. Antibiotic Prophylaxis for Patients With Newly Diagnosed Multiple Myeloma: Systematic Review and Meta-Analysis. Eur J Haematol (2020) 104(5):420-6. doi: 10.1111/ejh.13374

82. Valkovic T, Nacinovic AD, Petranovic D. Prophylactic Broad Spectrum Antibiotics as a New Anti-Myeloma Therapy. Med Hypotheses (2013) 81 (6):1137-40. doi: 10.1016/j.mehy.2013.10.021

83. Jego G, Bataille R, Geffroy-Luseau A, Descamps G, Pellat-Deceunynck C. Pathogen-Associated Molecular Patterns are Growth and Survival Factors for Human Myeloma Cells Through Toll-Like Receptors. Leukemia (2006) 20 (6):1130-7. doi: 10.1038/sj.leu.2404226

84. Bohnhorst J, Rasmussen T, Moen SH, Flottum M, Knudsen L, Borset M, et al. Toll-Like Receptors Mediate Proliferation and Survival of Multiple
Myeloma Cells. Leukemia (2006) 20(6):1138-44. doi: 10.1038/sj.leu. 2404225

85. Weber D, Jenq RR, Peled JU, Taur Y, Hiergeist A, Koestler J, et al. Microbiota Disruption Induced by Early Use of Broad-Spectrum Antibiotics is an Independent Risk Factor of Outcome After Allogeneic Stem Cell Transplantation. Biol Blood Marrow Transplant (2017) 23(5):845-52. doi: 10.1016/j.bbmt.2017.02.006

86. Shono Y, Docampo MD, Peled JU, Perobelli SM, Velardi E, Tsai JJ, et al. Increased GVHD-Related Mortality With Broad-Spectrum Antibiotic Use After Allogeneic Hematopoietic Stem Cell Transplantation in Human Patients and Mice. Sci Transl Med (2016) 8(339):339ra71. doi: 10.1126/scitranslmed.aaf2311

87. Abid H, Wu JF, Abid MB. Risk for Infections With Selinexor in Patients With Relapsed/Refractory Multiple Myeloma: A Systematic Review of Clinical Trials. Eur J Cancer (2021) 154:7-10.

88. D'Angelo C, Sudakaran S, Hematti P, Asimakopoulos F, Augustine C, Safdar $\mathrm{N}$, et al. Impact of Antibiotics on Gut Microbiota Diversity and the Results of a Prospective Dietary Assessment in Patients With Multiple Myeloma Undergoing Autologous Hematopoietic Stem Cell Transplantation. Blood (2019) 134(Supplement_1):4653-3. doi: 10.1182/blood-2019-123530

89. El Jurdi N, Filali-Mouhim A, Salem I, Retuerto M, Dambrosio NM, Baer L, et al. Gastrointestinal Microbiome and Mycobiome Changes During Autologous Transplantation for Multiple Myeloma: Results of a Prospective Pilot Study. Biol Blood Marrow Transplant (2019) 25(8):1511-9. doi: 10.1016/ j.bbmt.2019.04.007

90. Kusakabe S, Fukushima K, Maeda T, Motooka D, Nakamura S, Fujita J, et al. Pre- and Post-Serial Metagenomic Analysis of Gut Microbiota as a Prognostic Factor in Patients Undergoing Haematopoietic Stem Cell Transplantation. $\mathrm{Br}$ J Haematol (2020) 188(3):438-49. doi: 10.1111/bjh.16205

91. Bilinski J, Robak K, Peric Z, Marchel H, Karakulska-Prystupiuk E, Halaburda $\mathrm{K}$, et al. Impact of Gut Colonization by Antibiotic-Resistant Bacteria on the Outcomes of Allogeneic Hematopoietic Stem Cell Transplantation: A Retrospective, Single-Center Study. Biol Blood Marrow Transplant (2016) 22(6):1087-93. doi: 10.1016/j.bbmt.2016.02.009

92. Scheich S, Reinheimer C, Brandt C, Wichelhaus TA, Hogardt M, Kempf VAJ, et al. Clinical Impact of Colonization With Multidrug-Resistant Organisms on Outcome After Autologous Stem Cell Transplantation: A Retrospective Single-Center Study. Biol Blood Marrow Transplant (2017) 23(9):1455-62. doi: $10.1016 /$ j.bbmt.2017.05.016

Conflict of Interest: JB and GWB are the founders of the faecal microbiota bank and laboratory named the Human Biome Institute.

The remaining authors declare that the research was conducted in the absence of any commercial or financial relationships that could be construed as a potential conflict of interest.

Publisher's Note: All claims expressed in this article are solely those of the authors and do not necessarily represent those of their affiliated organizations, or those of the publisher, the editors and the reviewers. Any product that may be evaluated in this article, or claim that may be made by its manufacturer, is not guaranteed or endorsed by the publisher.

Copyright (๑) 2021 Jasiński, Biliński and Basak. This is an open-access article distributed under the terms of the Creative Commons Attribution License (CC BY). The use, distribution or reproduction in other forums is permitted, provided the original author(s) and the copyright owner(s) are credited and that the original publication in this journal is cited, in accordance with accepted academic practice. No use, distribution or reproduction is permitted which does not comply with these terms. 